\title{
Wavelet Network Model Based on Multiple Criteria Decision Making for Forecasting Temperature Time Series
}

\author{
Jian Zhang, Xiao-hua Yang, and Xiao-juan Chen \\ School of Environmental Sciences, Beijing Normal University, Beijing 100875, China \\ Correspondence should be addressed to Xiao-hua Yang; xiaohuayang@bnu.edu.cn
}

Received 24 October 2014; Revised 18 January 2015; Accepted 25 January 2015

Academic Editor: Hector Puebla

Copyright (C) 2015 Jian Zhang et al. This is an open access article distributed under the Creative Commons Attribution License, which permits unrestricted use, distribution, and reproduction in any medium, provided the original work is properly cited.

\begin{abstract}
Due to nonlinear and multiscale characteristics of temperature time series, a new model called wavelet network model based on multiple criteria decision making (WNMCDM) has been proposed, which combines the advantage of wavelet analysis, multiple criteria decision making, and artificial neural network. One case for forecasting extreme monthly maximum temperature of Miyun Reservoir has been conducted to examine the performance of WNMCDM model. Compared with nearest neighbor bootstrapping regression (NNBR), the probability of relative error smaller than $10 \%$ increases from $65.79 \%$ to $84.21 \%$ (forecast period $T=1$ ) and from $51.35 \%$ to $91.89 \%(T=2)$ by WNMCDM model. Similarly, the probability of relative error smaller than $20 \%$ increases from $84.21 \%$ to $97.37 \%(T=1)$ and from $81.08 \%$ to $91.89 \%(T=2)$ by WNMCDM model. Therefore, WNMCDM model is superior to NNBR model in forecasting temperature time series.
\end{abstract}

\section{Introduction}

Temperature time series are closely related to human life. The accurate prediction of the temperature time series offers important information for the city planning, land use, the design of civil project, and water resource management. Meteorological system is the result of the comprehensive effect of climate factors and human activity factors [1]. It is difficult for a single method to establish an effective model [2].

In recent years, wavelet analysis has become a research boom. It has huge advances in signal processing, image compress and encoding, tongue encoding, mode identification, and nonlinear science fields. Wavelet analysis has good multitime and scale features, which provides useful decompositions of original time series; so wavelet-transformed data improves other models the forecasting ability by capturing useful information on various resolution levels. The document [3] pointed out the potential applications of wavelet analysis to analyze temperature series. Jones and Moberg [4] studied the multiscale characteristics of temperature. Therefore, wavelet analysis has made great progress in the analysis of temperature time series.
Artificial neural network (ANN) has shown great ability in modeling and forecasting nonlinear and nonstationary time series in meteorology and water resources engineering due to its adaptive, self-organizing, self-learning ability. Campolo et al. [5] reported that their ANN model had better prediction accuracy and flexibility than statistical regression and simple conceptual models. Fan and Fu [6] presented an improved BP algorithm to optimize weights of neural network and achieved great prediction effect. In conclusion, ANN is a good method to predict temperature series.

Wavelet neural network model for predicting time series has become a hot study area since Zhang and Benveniste firstly proposed concepts and algorithms of wavelet neural network (WNN) and applied it for chaotic time series prediction [7]. The wavelet network model achieved good results in studying and predicting chaotic time series. Lv and Zhao [8] indicated that wavelet network method is more accurate than neural network from simulation results and can be effectively used in the prediction of nonlinear time series. Wang and Ding [9] revealed that wavelet network method could increase the forecasted accuracy and prolong the length time of prediction. But the hidden nodes are difficult to decide, and multiple criteria decision making can 
solve this problem. Wavelet network model based on multiple criteria decision making (WNMCDM) is firstly proposed in this paper. We will introduce the theory of WNMCDM model and prove the feasibility and accuracy by a practical case.

\section{WNMCDM Model}

Each part of wavelet network model based on multiple criteria decision making (WNMCDM) plays an importance role in forecasting time series. Wavelet analysis is used to determine cycle of temperature series and obtain high and low frequency components. Then artificial neural network is applied to predict future temperature by using above high and low frequency components. In the meantime, multiple criteria decision making is critical in determining the hidden nodes of ANN. The specific steps are shown in following context.

Step 1 (wavelet analysis). Discrete wavelet transform is selected to decompose and reconstruct the time series because observed time series in the real world are usually discrete, such as monthly runoff series and monthly temperature series $[10,11]$. We adopt common discrete wavelet transform a trous in this paper. Complex time sequence $Z_{t}(t=1,2, \ldots, n)$ is decomposed into different frequency blocks by [12]

$$
\begin{gathered}
C_{i}(t)=\sum_{k=-\infty}^{+\infty} h(k) C_{i-1}\left(t+2^{i} k\right), \\
W_{i}(t)=C_{i-1}(t)-C_{i}(t),
\end{gathered}
$$

where $h(k)$ is the discrete low-pass filter; in this paper, $B_{3}$ spline defined as $(1 / 16,1 / 4,3 / 8,1 / 4,1 / 16)$ is used [13]; $C_{i}(t), W_{i}(t)(i=1,2, \ldots, P)$ are background information (low frequency) and detail information (high frequency) and $C_{0}(t)=Z_{t} ; P$ is the scale which generally takes the natural logarithm of $n$. $\left\{W_{1}(t), W_{2}(t), \ldots, W_{p}(t), C_{p}(t)\right\}$ are called discrete wavelet transform with the resolution level $P$.

Step 2 (artificial neural network). Recent studies have shown that three-layer ANN network model can depict any complex nonlinear function, which basically solves the forecasting and simulation work. So three-layer neural network is also suitable for predicting temperature series. But input data needs to be standardized firstly by limiting to the range $[0,1]$. The input of BP network is $X=\left[W_{1}(t), W_{2}(t), \ldots, W_{P}(t), C_{P}(t)\right]^{T}$, and the number of nodes is $P+1$. The output is $Y=[Z(t+T)]$, and the number of nodes is 1 . Figure 1 shows the structure of ANN. Hidden layer nodes are determined by multiple criteria decision making by Step 3. Conjugate gradient momentum $\mathrm{BP}$ algorithm has been adopted in this paper.

Step 3 (multiple criteria decision making). In order to improve computational efficiency and accuracy, it is necessary to take root mean squared error (RMSE) and mean absolute error (MAE) and modified coefficient of efficiency (MCE) to determine accurate number of hidden nodes. MAE,

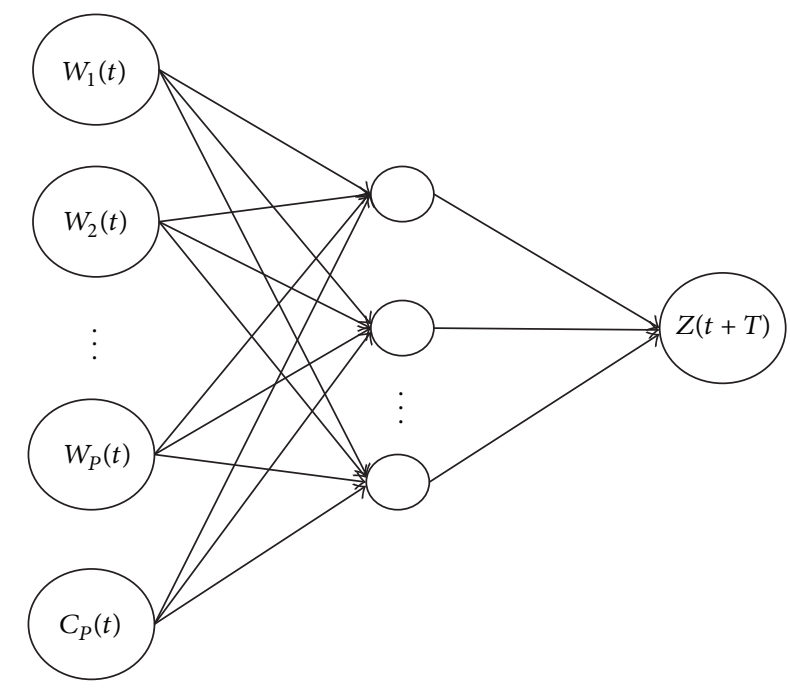

FIGURE 1: Structure diagram of ANN.

RMSE, and MCE are defined as follows. Low MAE and RMSE and high MCE mean better prediction effect [14]:

$$
\begin{aligned}
\text { MAE } & =\frac{1}{n} \sum_{i=1}^{n}\left|y_{i}-x_{i}\right|, \\
\text { RMSE } & =\sqrt{\frac{1}{n} \sum_{i=1}^{n}\left(y_{i}-x_{i}\right)^{2},} \\
\text { MCE } & =1-\frac{\sum_{i=1}^{n}\left|y_{i}-x_{i}\right|}{\sum_{i=1}^{n}\left|x_{i}-\bar{x}\right|} .
\end{aligned}
$$

\section{Applications in Extreme Temperature}

3.1. Study Area and Data. Miyun Reservoir is a large water conservancy that controls flood and supplies water for industrial and agricultural activities in region, which is a major source of urban water supply of Beijing. It has a total capacity of 4.375 billion $\mathrm{m}^{3}$ and controls $15788 \mathrm{~km}^{2}$ of river basin area [15]. Given the importance of Miyun Reservoir in supplying water for Beijing and the deterioration of climate change, it is vital to analyze the meteorological conditions. Therefore, the extreme monthly maximum temperature from 1989 to 2009 at Miyun Reservoir is applied to conduct the experiment. In order to facilitate research, we deliberately expanded ten times of the original sequence.

3.2. Wavelet Analysis. The original series is decomposed into $\left\{W_{1}(t), W_{2}(t), W_{3}(t), C_{3}(t)\right\}$ by a trous with the scale $P=3$. Figure 2 shows the wavelet decomposition sequences $\left\{W_{1}(t), W_{2}(t), W_{3}(t), C_{3}(t)\right\}$.

3.3. Extreme Monthly Maximum Temperature Forecast by WANN. Given two forecasting periods ( $T=1,2$ months), the first 176 months' data is used to train the model, the remaining 38 months for validation of $\mathrm{ANN}$, and the last 

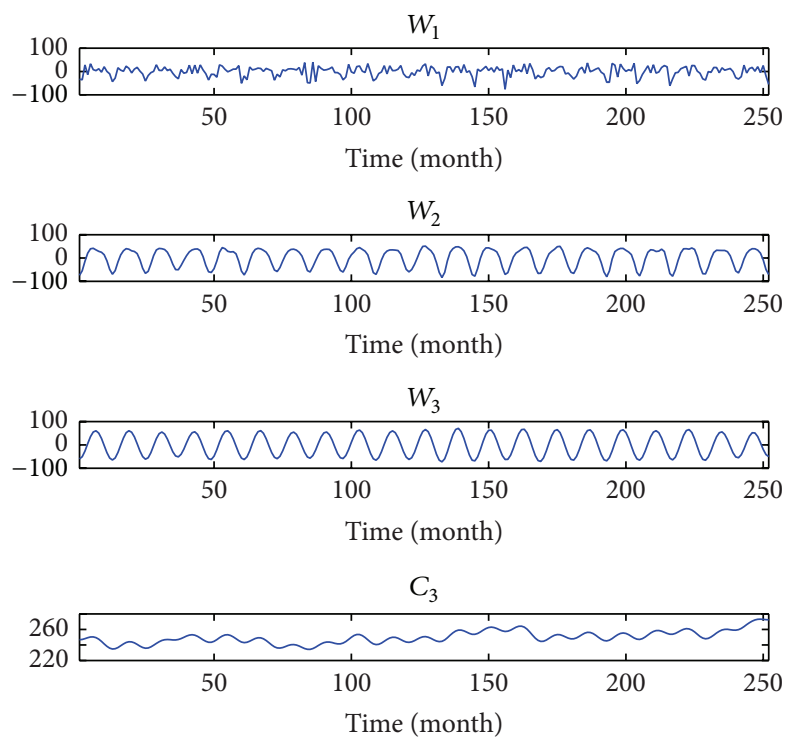

Figure 2: Wavelet decomposition sequences of extreme monthly maximum temperature in Miyun Reservoir.

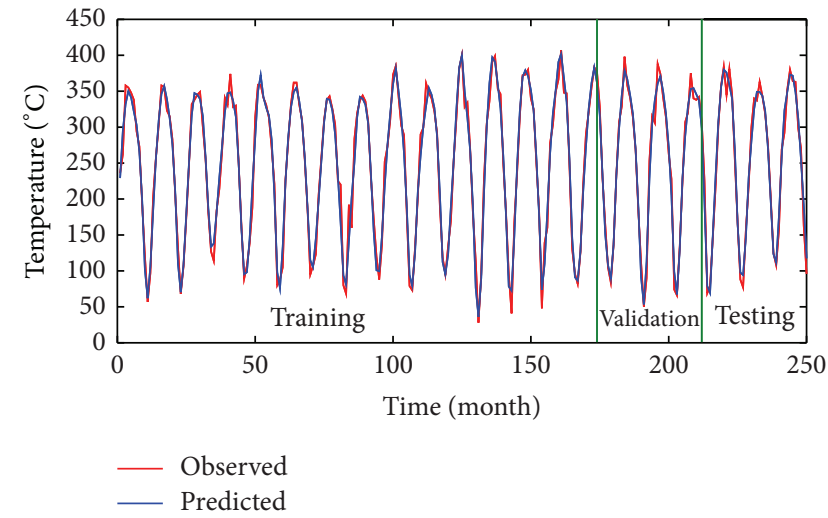

Figure 3: Comparison results $(T=1)$ between observed value and predicted value by ANN.

38 month for testing the model. Then scale number $P=3$; therefore the input data is $\left(\left[W_{1}(t), W_{2}(t), W_{3}(t), C_{3}(t)\right]\right)$ and the output is $Y=[Z(t+T)]$. We initially set the hidden layer nodes as 10 . So the structure of network is 4-10-1. The weight parameters of network are estimated by conjugate gradient momentum BP algorithm. Training times are 5000. Figure 3 shows the comparison results $(T=1)$ between observed values and predicted results by ANN.

3.4. Multiple Criteria Decision Making. In order to improve the prediction accuracy, multiple criteria decision making is used to revise hidden nodes of WANN model and reduce the uncertain part. Taking the hidden nodes from 5 to 10 , given two forecasting periods $(T=1,2)$, Table 1 shows that hidden nodes are 7 with higher MCE and lower MAE, RMSE.

3.5. Comparing to NNBR Model. The comparative analysis of testing value based on the extreme monthly maximum
TABLE 1: MCE, MAE, and RMSE results of different hidden nodes.

\begin{tabular}{lcccccc}
\hline \multirow{2}{*}{ Hidden nodes } & & $T=1$ & & & $T=2$ & \\
& MAE & RMSE & MCE & MAE & RMSE & MCE \\
\hline 5 & 11.49 & 14.13 & 0.87 & 10.64 & 13.04 & 0.88 \\
6 & 11.54 & 13.46 & 0.87 & 10.97 & 13.76 & 0.88 \\
7 & $\mathbf{1 0 . 2 5}$ & $\mathbf{1 2 . 1 4}$ & $\mathbf{0 . 8 9}$ & $\mathbf{1 0 . 6 4}$ & $\mathbf{1 2 . 9 9}$ & $\mathbf{0 . 8 8}$ \\
8 & 10.51 & 12.78 & 0.88 & 14.29 & 17.54 & 0.85 \\
9 & 10.13 & 12.43 & 0.89 & 11.71 & 14.68 & 0.87 \\
10 & 10.83 & 13.16 & 0.88 & 19.96 & 24.81 & 0.78 \\
\hline
\end{tabular}

TABLE 2: The comparative results of the extreme monthly maximum temperature by using WNMCDM and NNBR based monthly average temperature of Miyun Reservoir.

\begin{tabular}{|c|c|c|c|c|c|}
\hline \multirow{2}{*}{\multicolumn{2}{|c|}{$\begin{array}{c}\text { Model } \\
\text { Forecast period }\end{array}$}} & \multicolumn{2}{|c|}{ WNMBP } & \multicolumn{2}{|c|}{ NNBR } \\
\hline & & $T=1$ & $T=2$ & $T=1$ & $T=2$ \\
\hline \multirow{2}{*}{ Test } & Error $<10 \%$ & 84.21 & 91.89 & 65.79 & 51.35 \\
\hline & Error $<20 \%$ & 97.37 & 91.89 & 84.21 & 81.08 \\
\hline
\end{tabular}

temperature by using the WNMCDM model and NNBR model is displayed in Table 2. Figures 4(a) and 4(b) present the predicted results between the observed value and predicted value of last 38 months. It is obvious to see that the WNMCDM model has better prediction accuracy due to good forecasting results. Table 2 shows that the probability of relative error smaller than $10 \%$ could increase from $65.79 \%$ to $84.21 \%(T=1)$ and from $51.35 \%$ to $91.89 \%(T=2)$ by WNMCDM model; similarly, the probability of relative error smaller than $20 \%$ could increase from $84.21 \%$ to $97.37 \%$ $(T=1)$ and from $81.08 \%$ to $91.89 \%(T=2)$ by WNMCDM model.

\section{Conclusion}

In order to get the better prediction of temperature time series, three steps are carried out. The first step is using wavelet analysis to get decomposed sequences. The second step is applying artificial neural network to predict temperature. At last, multiple criteria decision making is critical in determining the hidden nodes of ANN and reduce their uncertain ingredients. WNMCDM model is superior to NNBR model in predicting extreme monthly maximum temperature of Miyun Reservoir because the probability of relative error smaller than $10 \%$ could increase from $65.79 \%$ to $84.21 \%(T=1)$ and from $51.35 \%$ to $91.89 \%(T=2)$ by WNMCDM model; similarly, the probability of relative error smaller than 20\% could increase from $84.21 \%$ to $97.37 \%$ $(T=1)$ and from $81.08 \%$ to $91.89 \%(T=2)$ by WNMCDM model.

However, many factors affect the temperature time series. Further study of WNMBP model about meteorological forecast will be implemented involving climate change, the impact of human activities, and other factors. In this way, the prediction of time series will be more reliable and more accurate. 


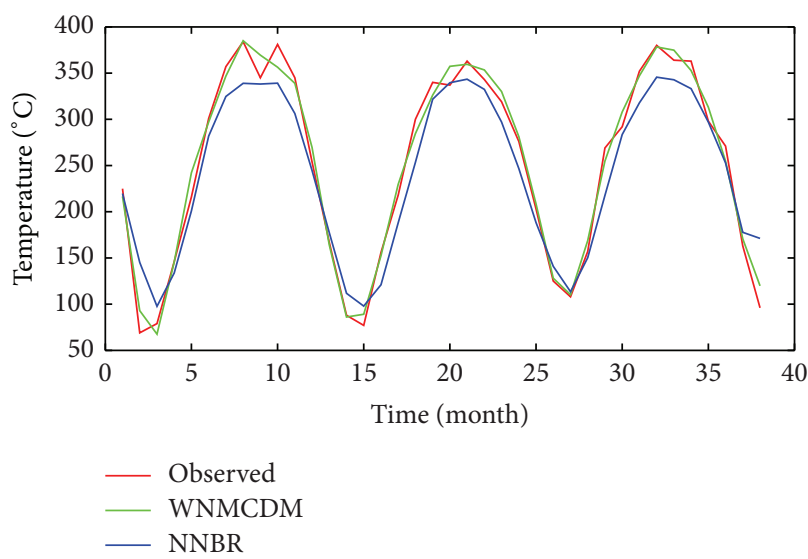

(a)

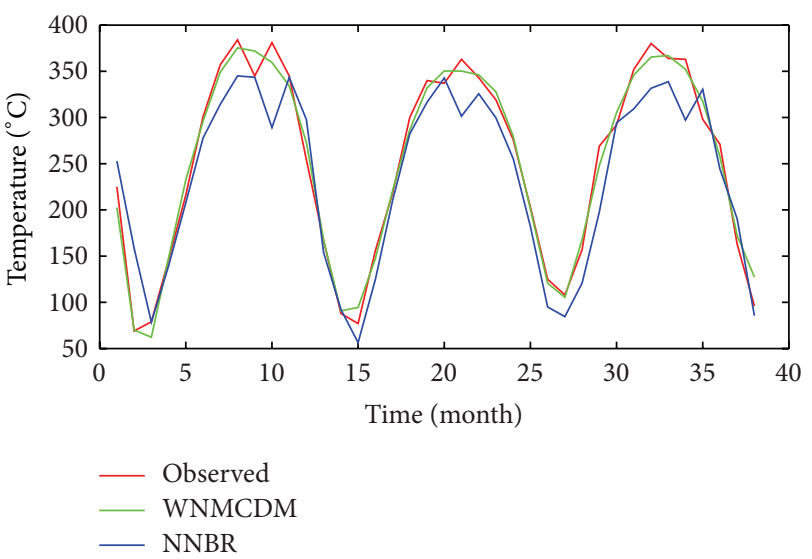

(b)

FIGURE 4: (a) Comparison results $(T=1)$ between observed and predicted value by WNMCDM and NNBR model. (b) Comparison results $(T=2)$ between observed and predicted value by WNMCDM and NNBR model based on the extreme monthly maximum temperature of Miyun Reservoir.

\section{Conflict of Interests}

The authors declare that there is no conflict of interests regarding the publication of this paper.

\section{Acknowledgments}

This work was supported by the Project of National Natural Science Foundation of China (nos. 50939001, and 51379013), the National Basic Research Program of China (no. 2010CB951104), the Funds for Creative Research Groups of China (no. 51121003), the Specialized Research Fund for the Doctoral Program of Higher Education (no. 20100003110024), and the Program for Changjiang Scholars and Innovative Research Team in University (no. IRT0809).

\section{References}

[1] B. Bošnjaković, "Geopolitics of climate change: a review," Thermal Science, vol. 16, no. 3, pp. 629-654, 2012.

[2] Y. P. Huang and T. M. Yu, "The hybrid grey-based models for temperature prediction," IEEE Transactions on Systems, Man, and Cybernetics, Part B: Cybernetics, vol. 27, no. 2, pp. 284-292, 1997.

[3] T. W. Kim and J. B. Valdés, "Nonlinear model for drought forecasting based on a conjunction of wavelet transforms and neural networks," Journal of Hydrologic Engineering, vol. 8, no. 6, pp. 319-328, 2003.

[4] P. D. Jones and A. Moberg, "Hemispheric and large-scale surface air temperature variations: an extensive revision and an update to 2001," Journal of Climate, vol. 16, no. 2, pp. 206-223, 2003.

[5] M. Campolo, P. Andreussi, and A. Soldati, "River flood forecasting with a neural network model," Water Resources Research, vol. 35, no. 4, pp. 1191-1197, 1999.

[6] C. N. Fan and D. S. Fu, "Using neural network to forecast meteorological temperature," Computer Applications and Software, vol. 21, no. 3, pp. 108-110, 2004.
[7] Q. Zhang and A. Benveniste, "Wavelet networks," IEEE Transactions on Neural Networks, vol. 3, no. 6, pp. 889-898, 1992.

[8] S. P. Lv and Y. M. Zhao, "The method and application of time series prediction based wavelet neural network," Journal of Harbin Engineering University, vol. 25, no. 2, pp. 180-182, 2004.

[9] W. S. Wang and J. Ding, "Wavelet network model and its application to the prediction of hydrology," Nature and Science, vol. 1, no. 1, pp. 67-71, 2003.

[10] P. Kumar and G. E. Foufoula, "A multicomponent decomposition of spatial rainfall fields: segregation of large and small-scale features using wavelet transforms," Water Resources Research, vol. 29, no. 8, pp. 2515-2532, 1993.

[11] C. Di, X. Yang, and X. Wang, "A four-stage hybrid model for hydrological time series forecasting," PLoS ONE, vol. 9, no. 8, Article ID e104663, 2014.

[12] S. Mallat, A Wavelet Tour of Signal Processing, Academic Press, San Diego, Calif, USA, 1998.

[13] W. S. Wang, J. L. Jin, and Y. Q. Li, "Prediction of inflow at three Gorges dam in Yangtze River with wavelet network model," Water Resources Management, vol. 23, no. 13, pp. 2791-2803, 2009.

[14] X. H. Yang, D. X. She, Z. F. Yang, Q. H. Tang, and J. Q. Li, "Chaotic bayesian method based on multiple criteria decision making (MCDM) for forecasting nonlinear hydrological time series," International Journal of Nonlinear Sciences \& Numerical Simulation, vol. 10, no. 11, pp. 1595-1610, 2009.

[15] X. Q. Duan, L.-J. Hao, and F.-F. Luan, "Analysis of the characteristics of the rainfall and runoff in the basin of the Miyun reservoir," Beijing Water, vol. 1, pp. 38-41, 2013. 


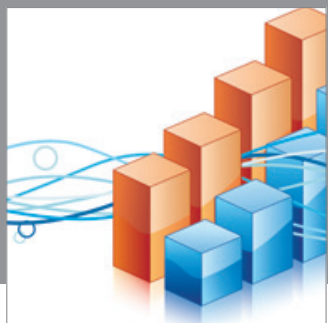

Advances in

Operations Research

mansans

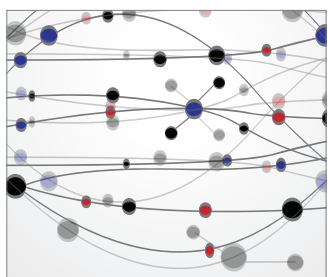

The Scientific World Journal
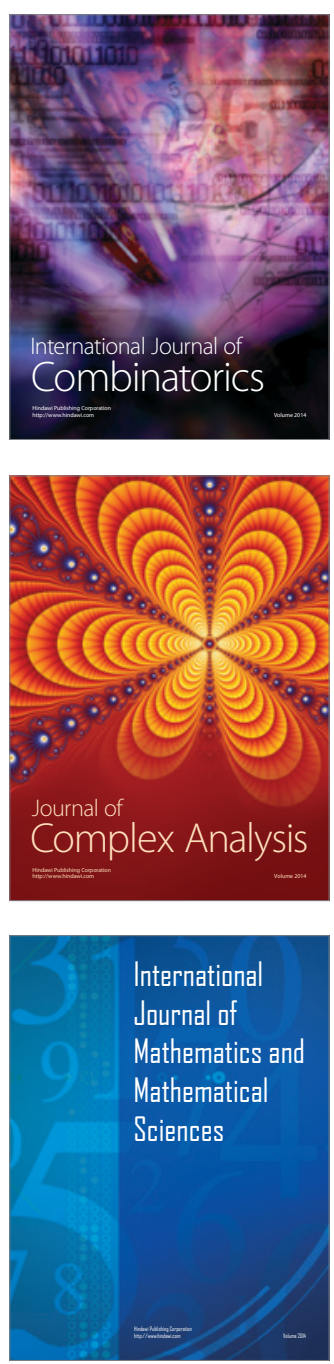
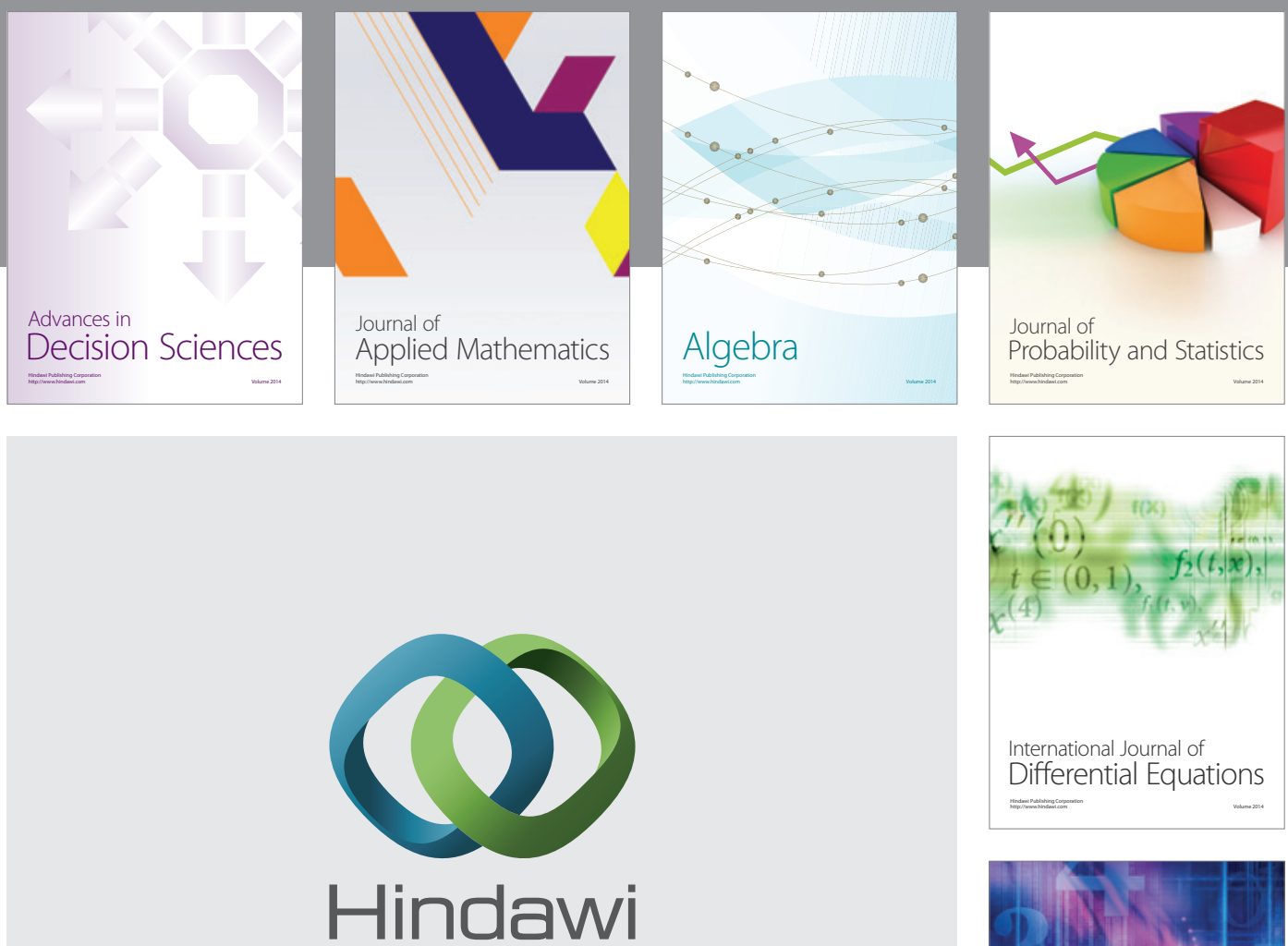

Submit your manuscripts at http://www.hindawi.com
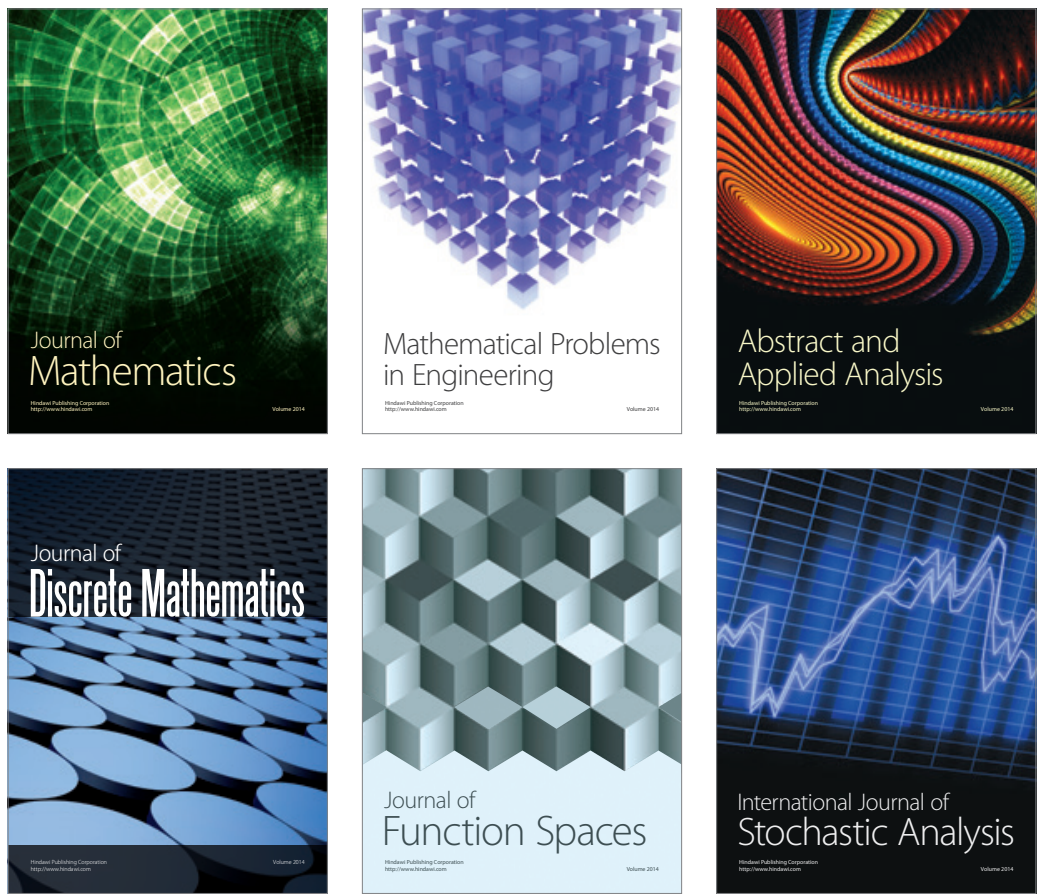

Journal of

Function Spaces

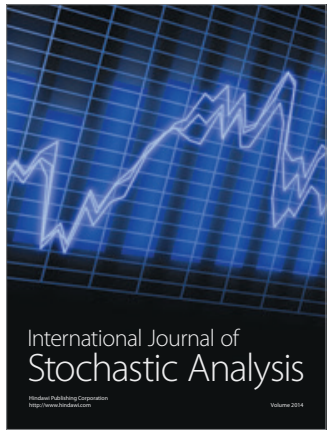

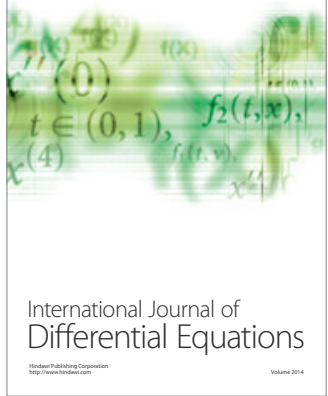
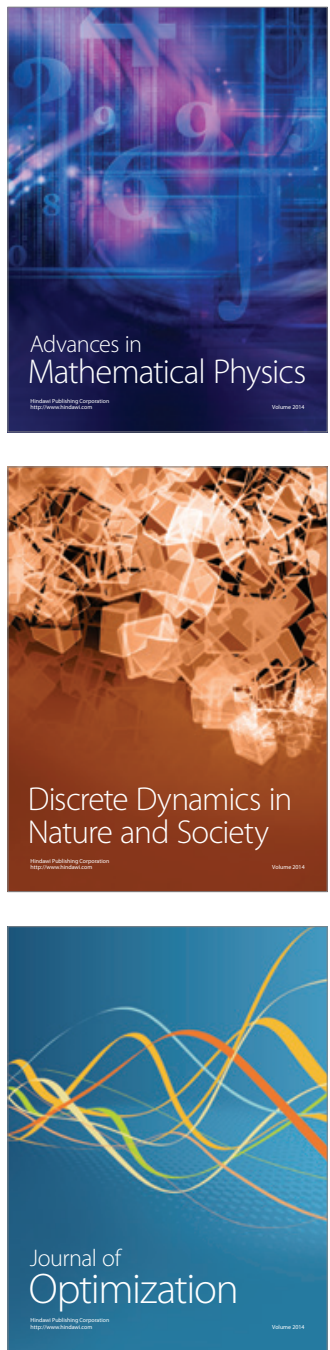